PÁGINA DEL EDITOR

\title{
Barreras para el desarrollo de centros de investigacion
}

\section{Barriers to the development of research centers}

\author{
1,35tefany Cielo Oporto Zenteno, ${ }^{2,3}$ Danae Carelis Davila Espinoza
}

E stamos de acuerdo en que la investigación es crucial para el crecimiento académico, profesional e institucional de cualquier universidad, lo que nos hace concluir que, el desarrollo científico con la creación de sociedades y revistas científicas, así como su apoyo y sustento, son pilares fundamentales para el progreso de una institución académica.

En comparación a otros países latinoamericanos, Bolivia tiene un mínimo de producción científica, sin existir punto de comparación con países de primer mundo, este hecho debería llevar a que las entidades facultativas se pregunten el por qué de esta escasez. Muchas investigaciones que intentan explicar la falta de producción, concluyen que la causa es la "falta de interés" pero muy pocos identifican la ausencia de centros formadores y la falta de apoyo instructivo formativo impartido por su propia universidad.

Realizando una encuesta a varias sociedades científicas y revistas científicas estudiantiles de la Universidad Mayor de San Simón, se encontró que más de $90 \%$ de estudiantes encuestados sienten una falta de apoyo académico por parte de su propia facultad es decir, pocos son los profesionales docentes que se prestan desinteresadamente a colaborar con enseñanza a los estudiantes pertenecientes de estas sociedades. Analizando también otras respuestas abiertas a los estu- diantes expresaron repetidas veces: la falta de reconocimiento como jóvenes investigadores, déficit económico, burocracia incluyendo el infundado número de requisitos o la negativa injustificada que impiden el desarrollo de investigación dentro de instituciones aun estando afiliadas a la universidad, menosprecio falta de apoyo moral e indiferencia de autoridades académicas con el trabajo de su sociedad, entre otros.

Sobrellevando los obstáculos y desafíos puestos por las mismas entidades universitarias, por años se ha visto que las sociedades $y$ revistas científicas se han autosustentado académica y económicamente, con la colaboración de profesionales que únicamente por amor a la ciencia y esperanza en los futuros investigadores se prestan desinteresadamente al llamado de estudiantes miembros de estas instituciones.

Para solucionar un problema se debe "quitarlo desde la raiz", entonces si la "raíz" es la falta de interés, este debe ser subsanado por sus guías es decir docentes, desde el inicio de la formación académica, ya que al tratarse de personas que un estudiante en formación tiene como referencia considerara este área como parte de su formación profesional y no una tarea extra y ajena a la academia.

Plantear una estrategia que cree una conexión directa institucional - estudiantil, a fin que los estudiantes interesados en desarrollar ciencia tengan un camino accesible para alcanzar sus propósitos, permitiendo un crecimiento institucional y creando bases que fomentaran a que la siguiente generación de investigadores continúe, logrando así un círculo virtuoso.

La universidad es el hogar de la ciencia, la preparación de investigadores y de nuevas mentes maestras, aquellas que serán la generación que definirá la manera en que cambiara el mundo y pondrán en marcha el país, por ello es importante evitar que la inspiración, motivación y esperanza de esta generación se ausente, trabajando juntos para el ascenso científico.

1 Stefany Cielo Oporto Zenteno

Editor Revista Científica Ciencia Médica. ORCID ID: https://orcid.org/0000-00028577-0471

${ }^{2}$ Danae Carelis Davila Espinoza Editor Asociado Revista Científica Ciencia Médica.

ORCID ID: https://orcid.org/0000-00020877-5041

${ }^{3}$ Sociedad Científica de Estudiantes de Medicina, Facultad de Medicina “Dr.

Aurelio Melean", Universidad Mayor de San Simón. Cochabamba-Bolivia.

Correspondencia a: Stefany Cielo Oporto Zenteno oportocielo8@gmail.com

Citar como:

Oporto Zenteno SC, Davila Espinoza DC, Barreras para el desarrollo de centros de investigacion Rev Cient Cienc Med. 2021; 24(1): 3 